\title{
Impact of changes in grain size and pore space on the hydraulic conductivity and spectral induced polarization response of sand
}

\author{
K. Koch ${ }^{1}$, A. Kemna ${ }^{2}$, J. Irving ${ }^{1}$, and K. Holliger ${ }^{1}$ \\ ${ }^{1}$ Institute of Geophysics, University of Lausanne, 1015 Lausanne, Switzerland \\ ${ }^{2}$ Department of Geodynamics and Geophysics, University of Bonn, 53115 Bonn, Germany
}

Received: 12 August 2010 - Published in Hydrol. Earth Syst. Sci. Discuss.: 24 August 2010

Revised: 20 May 2011 - Accepted: 29 May 2011 - Published: 9 June 2011

\begin{abstract}
Understanding the influence of pore space characteristics on the hydraulic conductivity and spectral induced polarization (SIP) response is critical for establishing relationships between the electrical and hydrological properties of surficial unconsolidated sedimentary deposits, which host the bulk of the world's readily accessible groundwater resources. Here, we present the results of laboratory SIP measurements on industrial-grade, saturated quartz samples with granulometric characteristics ranging from fine sand to fine gravel. We altered the pore space characteristics by changing (i) the grain size spectra, (ii) the degree of compaction, and (iii) the level of sorting. We then examined how these changes affect the SIP response, the hydraulic conductivity, and the specific surface area of the considered samples. In general, the results indicate a clear connection between the SIP response and the granulometric as well as pore space characteristics. In particular, we observe a systematic correlation between the hydraulic conductivity and the relaxation time of the Cole-Cole model describing the observed SIP effect for the entire range of considered grain sizes. The results do, however, also indicate that the detailed nature of these relations depends strongly on variations in the pore space characteristics, such as, for example, the degree of compaction. This underlines the complexity of the origin of the SIP signal as well as the difficulty to relate it to a single structural factor of a studied sample, and hence raises some fundamental questions with regard to the practical use of SIP measurements as site- and/or sample-independent predictors of the hydraulic conductivity.
\end{abstract}

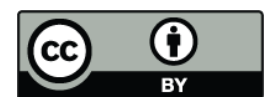

Correspondence to: $\mathrm{K}$. Koch (krskoch@gmail.com)

\section{Introduction}

Knowledge of the distribution of the hydraulic conductivity within an aquifer is a key prerequisite for reliable predictions of groundwater flow and contaminant transport. This information is in turn critical for the effective protection, remediation, and sustainable management of increasingly scarce and fragile groundwater resources in densely populated and/or highly industrialized regions throughout the world. To this end, geophysical constraints with regard to aquifer structure and the distribution of hydraulic parameters are considered to be particularly valuable. The primary reasons for this are that geophysical methods are less expensive than other direct investigation methods and non-invasive in nature, and that they have the potential to bridge an inherent gap which exists in terms of spatial resolution and coverage between traditional hydrogeological methods such as core analyses and tracer or pumping tests (e.g., Rubin and Hubbard, 2005; Koch et al., 2009).

Although standard geophysical techniques cannot in general provide any direct information regarding the hydraulic conductivity in the subsurface, there are a number of more specialized approaches that exhibit a more-or-less direct sensitivity to this important parameter (e.g., Slater, 2007; Holliger, 2008). In particular, induced polarization (IP) and spectral induced polarization (SIP) measurements represent promising methods. A clear link between hydrological properties and IP/SIP parameters has been empirically documented by various studies (e.g., Börner et al., 1996; Slater and Lesmes, 2002; Kemna et al., 2004; Binley et al., 2005; Hördt et al., 2007; Slater, 2007). However, recent studies by Binley et al. (2010), Kruschwitz et al. (2010), and Titov et al. (2010) also point out that the detailed nature and origin of such linkages remain enigmatic. In particular, it is not clear how basic changes in the pore space and/or grain size characteristics affect the SIP response and its relation to the hydraulic conductivity.

Published by Copernicus Publications on behalf of the European Geosciences Union. 
In this paper, we address the question of whether characteristics related to grain or to pore size affect most the SIP relaxation time. We do so through SIP measurements on saturated industrial-grade granular quartz samples with effective grain sizes ranging from fine sand to fine gravel. The pore space and grain size characteristics of the original samples are modified through compaction and sieving, while the chemistry of the saturating pore fluid is kept constant in order to examine the impact of structural changes on the SIP response. We express the impact of these variations with regard to the hydraulic conductivity and subsequently discuss its relation with the recorded SIP response, in particular the determined relaxation time.

In the following, we first describe the basic principles of electrochemical polarization and relaxation, followed by our experimental setup, the granulometric properties of the samples used in this study, and the basic data analysis methodology.

\section{Electrochemical polarization and electrical relaxation}

The IP-type polarization of non-metallic minerals is generally referred to as interface or membrane polarization (Marshall and Madden, 1959; Vinegar and Waxman, 1984) and takes place in the lower frequency range up to the MaxwellWagner effect (Maxwell, 1892; Wagner, 1914; Chen and Or, 2006). In the absence of metallic conductors, such as ore minerals or graphite, IP phenomena are commonly associated with polarization effects related to a polarized electrical double layer (EDL). The EDL schematically describes the organization of ionic charges at the interface between solid and fluid and was first introduced by Helmholtz in the mid19th century. The inner layer is given by the typically negatively charged mineral surface attracting positively charged ions contained in the pore fluid to form the supposedly firmly attached Stern layer (Stern, 1924). Beyond the Stern layer, positively charged ions continue to be attracted by the negatively charged mineral surface, but at the same time are repelled by each other and the Stern layer. The resulting dynamic equilibrium is referred to as the diffuse layer and represents the transition zone between the Stern layer and the neutral part of the pore water. The individual elements of the diffuse layer are hence in equilibrium with the neutral part of the pore water as is the basis for the concentration profiles of anions and cations from the Boltzmann distributions derived via the equality of the electrochemical potentials between any distance in the diffuse layer and infinity (e.g., Revil and Glover, 1997). The EDL provides the conceptual background for the electrochemical processes considered to be responsible for the observed SIP response. Today's conceptual understanding of the origin of the SIP response is strongly based on the work of Schwarz (1962) and his interpretation of the polarization effect as a result of the redistribution of counter-ions surrounding spherical particles in suspension. He describes the system as counterions on the surface of a highly-charged colloidal particle which are strongly bound by electrostatic attraction. In order to escape from the surface into the free solution, they have to overcome a high potential barrier. Along the surface, however, they can be moved much more easily. Thus they will be moved tangentially by an external field, polarizing the ion atmosphere and inducing an electric dipole moment of the particle. Schwarz' model, therefore, comprises the entire double layer, while the source process is allocated within the tangential movement of a single layer of counterions, which in turn effects the ion atmosphere around the particle. The only geometric factor involved in this model is the size of the sphere. Translating this geometrically simple analytical model to texturally complex porous media is not evident, as it simply is too strong a simplification. The commonly applied models are based on polarization of the diffuse layer in the pore system (e.g., Dukhin and Shilov, 1974), where Titov et al. (2004), amongst others, attempted to provide a visualization of the two basic concepts with regard to the origin of the SIP effect in porous media by linking the relaxation length scale to either granular or capillary models. A number of studies have tried to gain further insight into these matters by attributing the polarization to the EDL surrounding the individual grains (e.g., Lesmes and Morgan, 2001) and to excesses and deficiencies in ion concentrations along pore throats (e.g., Titov et al., 2002). It is only based on the recent work of Leroy et al. (2008), Leroy and Revil (2009), Jougnot et al. (2010), and in particular Revil and Florsch (2010) that a more and more refined picture of polarization processes taking place in the Stern layer has started to emerge. Indeed, this work provides strong evidence to suggest that the Stern layer is potentially the region where the most important processes associated with the observed SIP response take place. In virtually all of these studies, the relaxation time or relaxation frequency has been theoretically related to a certain length scale representing either the grain radius (Schwarz, 1962) or a pore length scale (Kormiltsev, 1963).

With regard to the observed electrical relaxation, a number of workers have found consistently good correlations between hydraulic conductivity and the Cole-Cole time constant for a range of geological materials (e.g., Binley et al., 2005; Kemna et al., 2005; Tong et al., 2006; Zisser et al., 2010). Pelton et al. (1978) were arguably the first to illustrate the adequacy of Cole-Cole-type models (Cole and Cole, 1941) for phenomenological description of the monitored SIP responses (e.g., Vanhala, 1997; Dias, 2000). Recently, Revil and Florsch (2010) supplied a corresponding theoretical justification, which is based on the polarization of the Stern layer and supported by the results of several recent studies (Leroy et al., 2007; Jougnot et al., 2010; Schmutz et al., 2010). 


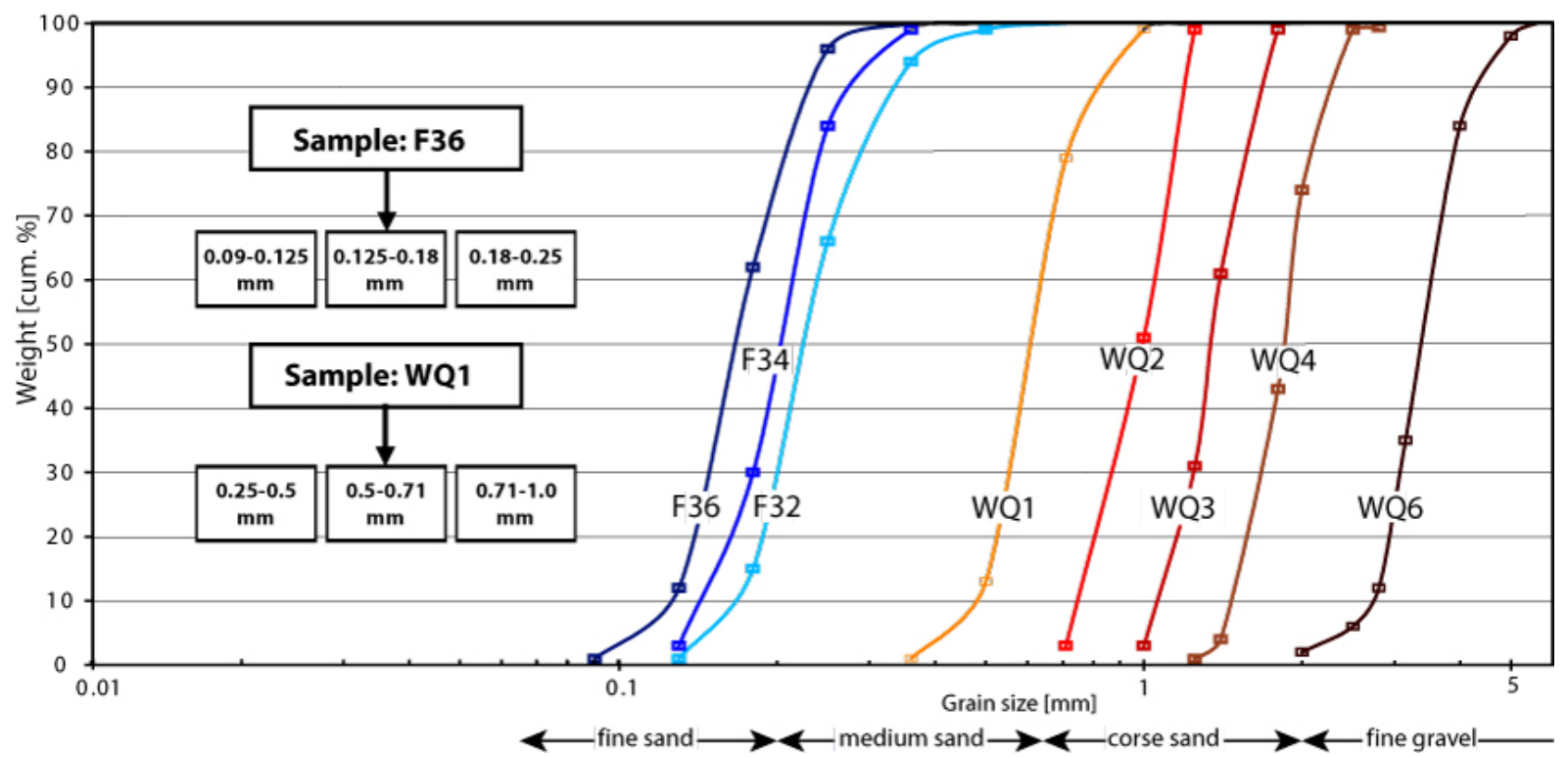

Fig. 1. Grain size distribution curves for the different samples considered in this study. Left: Six sieved fractions originating from sands F36 and WQ1. Right: Grain size distributions of unsieved industrial granular quartz samples. See also Table 1.

Table 1. Table of the original, unsieved samples and their effective and medium grain sizes $d_{10}$ and $d_{50}$, respectively, and their level of sorting as defined by $d_{60} / d_{10}$.

\begin{tabular}{lllllllll}
\hline & F36 & F34 & F32 & WQ1 & WQ2 & WQ3 & WQ4 & WQ6 \\
\hline Aggregate & Fine & Medium & Medium & Medium & Coarse & Coarse & Coarse & Fine \\
class & sand & sand & sand & sand & sand & sand & sand & gravel \\
$d_{10}[\mathrm{~mm}]$ & 0.12 & 0.14 & 0.16 & 0.47 & 0.75 & 1.06 & 1.46 & 2.7 \\
$d_{50}[\mathrm{~mm}]$ & 0.17 & 0.21 & 0.23 & 0.62 & 0.99 & 1.35 & 1.84 & 3.41 \\
$d_{60} / d_{10}$ & 1.50 & 1.57 & 1.50 & 1.38 & 1.40 & 1.31 & 1.31 & 1.33 \\
\hline
\end{tabular}

\section{Experimental procedure and data analysis}

We have performed laboratory-based measurements on water-saturated industrial-grade granular quartz samples over a very broad range of average grain diameters from fine sand to fine gravel (Table 1, Fig. 1). For these measurements, the pore space characteristics of the samples were modified by varying (i) the grain size, (ii) the degree of compaction, and (iii) the degree of sorting. We then examined how these changes affected the hydraulic conductivity, specific surface area, and the SIP response of the considered samples. Compaction was achieved through handheld multidirectional vibration. The shaking was continued until the sample volume stabilized at $90 \%$ of its original volume. Sieving the sands F36 and WQ1 provided extremely well sorted samples of grain sizes: $0.09-0.125 \mathrm{~mm}, 0.125-0.18 \mathrm{~mm}, 0.18-0.25 \mathrm{~mm}$, $0.25-0.5 \mathrm{~mm}, 0.5-0.71 \mathrm{~mm}$, and $0.71-1.0 \mathrm{~mm}$.

Measurements of the hydraulic conductivity were made using the constant head method. The porosity $\phi$ was determined by weighing the saturated samples and thus determining its density given as $\rho_{\text {total }}=(1-\phi) \rho_{\mathrm{SiO}_{2}}+\phi \rho_{\mathrm{H}_{2} \mathrm{O}}$ where $\rho_{\text {total }}$ is the inferred density of weighted sample and $\rho_{\mathrm{SiO}_{2}}$ and $\rho_{\mathrm{H}_{2} \mathrm{O}}$ are the a priori known densities of quartz and water, respectively. Specific surface area analysis was undertaken through the use of laser diffractometry. Specific surface measurements based on laser diffraction methods aim at estimating the grain diameters through the assumption of a specific geometrical form factor of the grain, which in our case is spherical. The signal of the diffracted light from a measured grain is thus fitted to that of an equivalent sphere. Hence, specific surface area measurements of this type provide us with the so-called geometric surface area of a grain, which is equal to the surface of the equivalent sphere. The corresponding specific surface area, given by the surface of this equivalent sphere divided by its volume, does therefore neither account for the small-scale grain surface roughness nor for the porosity. For a granulometrically heterogeneous sample, this parameter thus represents a combined measure 


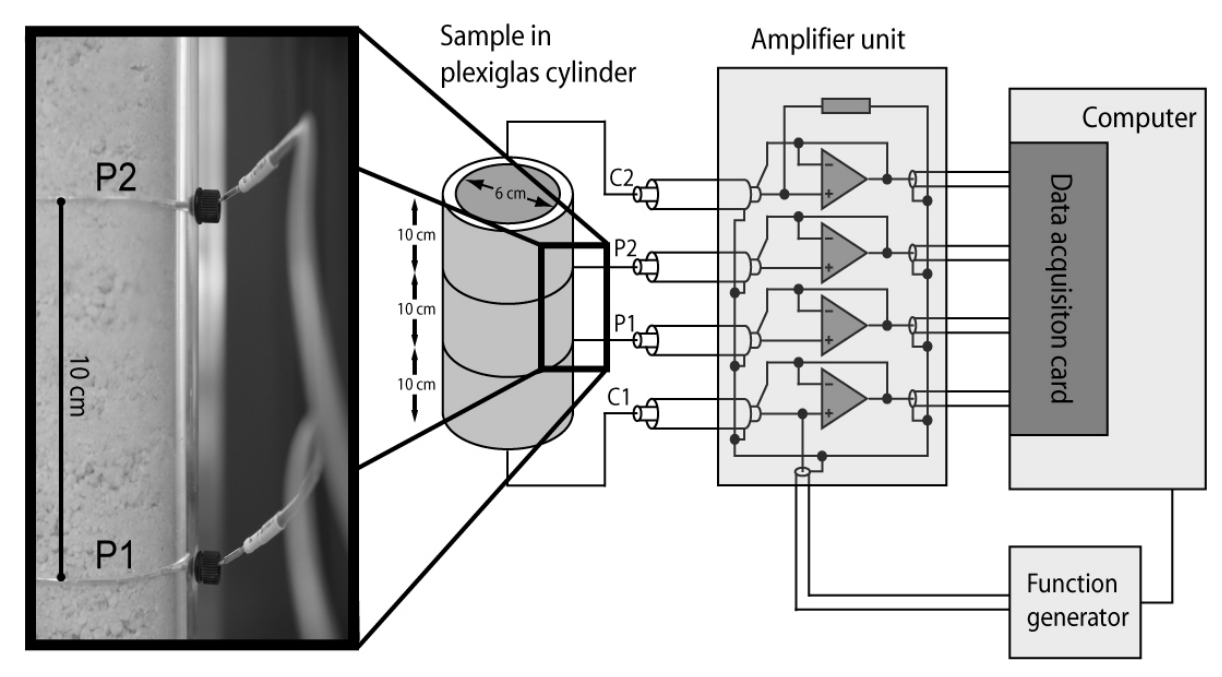

Fig. 2. Schematic illustration of the high-sensitivity impedance spectrometer used for the SIP measurements presented in this study. The sample holder corresponds to a plexiglas cylinder with an inner diameter of $6 \mathrm{~cm}$ and a length of $30 \mathrm{~cm}$. The current electrodes, $\mathrm{C} 1$ and $\mathrm{C} 2$, are made of circular porous bronze plates with a diameter of $6 \mathrm{~cm}$ and are located at the top and bottom of the sample holder. The potential electrodes, P1 and P2, correspond to rings made of silver wire located at a distance of $10 \mathrm{~cm}$ from the top and bottom of the measurement cylinder. These silver wires are embedded in grooves to keep them outside the actual sample and the electric field associated with the current electrodes and thus to minimize electrical polarization effects. Adapted from Zimmermann et al. (2008).

of sorting and grain size of the overall distribution. The SIP measurements were conducted over a frequency range from $1 \mathrm{mHz}$ to $45 \mathrm{kHz}$ and electrical conductivities of $\sim 60 \mu \mathrm{S} / \mathrm{cm}$ and $\sim 300 \mu \mathrm{S} / \mathrm{cm}$ were considered for the saturating pore fluids. In this context, it is important to note that in general the signal-to-noise ratio of the observed phase spectra improved significantly with decreasing electrical conductivity of the pore fluid. This is due to the fact that the SIP phase spectrum essentially corresponds to the ratio of the complex and real parts of the electrical conductivity and thus reflects the increasing relative importance of the complex surface conductivity processes taking place in the EDL at low conductivities of the saturating pore fluid. The SIP measurements were carried out using the highly sensitive impedance spectrometer for weakly polarizeable media developed by Zimmermann et al. (2008). The device is based on the four-point measurement method, and allows phase measurements in the required frequency range with an accuracy better than $0.1 \mathrm{mrad}$. Our experimental setup was designed to minimize polarization of the electrode materials. The cylinder holding the sample has a length of $30 \mathrm{~cm}$ and a diameter of $6 \mathrm{~cm}$. The current electrodes, consisting of porous bronze plates with an effective pore diameter of $15 \mu \mathrm{m}$, form the upper and lower boundaries of the sample volume. The potential electrodes correspond to rings made of silver wire placed into grooves along the inner wall of the 30-cm-long measurement cylinder (Fig. 2). These grooves are located at a distance of $10 \mathrm{~cm}$ from either end of cylinder. This results in an equi-distant spacing of $10 \mathrm{~cm}$ between individual electrodes and a geometric factor given as $k=r^{2} \pi / 1$ with $r$ and $l$ denoting the radius of the measurement cylinder and the distance between the potential electrodes, respectively. Fixing the potential electrodes into grooves aims at avoiding polarization effects by keeping the silver wire outside of the electric field associated with the current electrodes. The corresponding electrical connection to the electrodes is naturally provided through the conducting pore fluid, which fully saturates the sample filling the cylinder (Zimmermann et al., 2008). The sample's response to the applied current is recorded and provides information about the real and imaginary parts of the electrical resistivity.

To analyze the measured complex resistivity data, we first fit them using the so-called Cole-Cole model (Cole and Cole, 1941) given by

$$
\rho(\omega)=\rho_{0}\left[1-m\left(1-\frac{1}{1+(i \omega \tau)^{c}}\right)\right],
$$

where $\omega$ denotes angular frequency, $\rho_{0}$ the low-frequency asymptote, or direct-current value, of the electrical resistivity $\rho(\omega), m$ the chargeability, $c$ the Cole-Cole exponent, $\tau$ the time constant or relaxation time, and $i=\sqrt{-1}$. The chargeability $m$ describes the magnitude of the polarization effect, the Cole-Cole exponent $c$ determines the width of the peak of the phase curve described by Eq. (1), and the time constant $\tau$ is related to the location of this peak in the frequency band (e.g., Lesmes and Friedman, 2005; Cosenza et al., 2009, p. 573). The exponent $c$ typically takes values in the range from $\sim 0.2$ to $\sim 0.7$. Here, smaller values of $c$ are associated with broader resonance peaks. In a number of previous laboratory-based SIP studies, relaxation time has been found to exhibit a more-or-less clear correlation with the hydraulic conductivity (e.g., Pape and Vogelsang, 1996; Kemna 


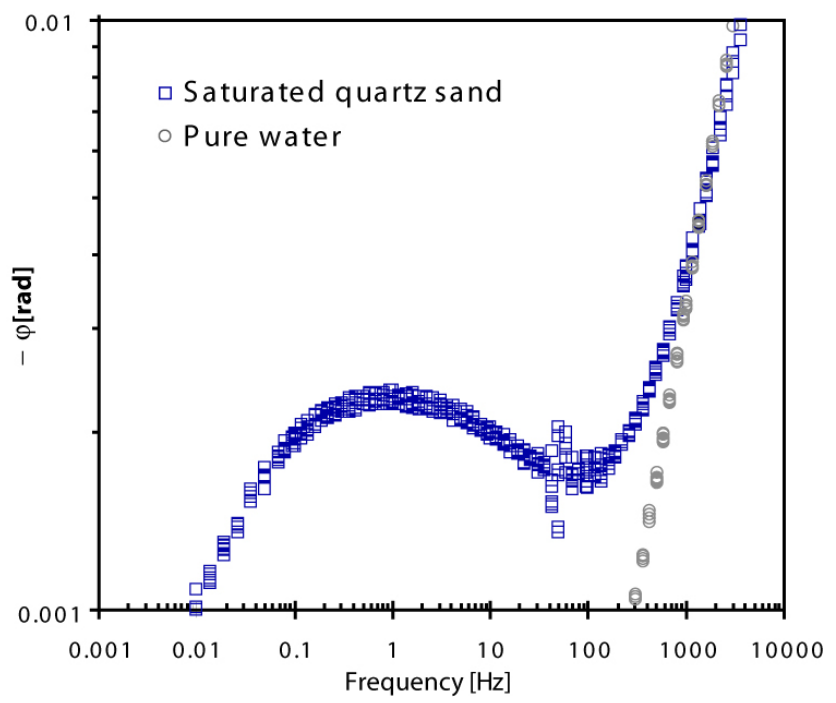

Fig. 3. Example of phase spectra obtained from SIP measurements on a saturated sample of sand F36 (Table 1) as well as on the corresponding saturating pore water alone. The latter had an electrical conductivity of $\sim 60 \mu \mathrm{S} / \mathrm{cm}$. Note the negative scale for the phase.

et al., 2005; Binley et al., 2005). To deal with the strong non-linearity and non-uniqueness of the inverse problem of estimating Cole-Cole model parameters that fit the measured frequency-domain SIP data, we use a Markov chain Monte Carlo (McMC) inversion approach in this study. With standard Monte Carlo parameter estimation methods, random sets of parameter values are generated and then either accepted or rejected based on how well they allow us to fit the measured data. McMC importance sampling intensifies the formerly completely random sampling in more "probable" areas of the model space, which allows for increased efficiency of the procedure. Sets of feasible model parameters are generated with a frequency according to their probability of occurrence. The inversion procedure is based on the work of Mosegaard and Tarantola (1995). For a detailed description of the application to SIP data, and a comparison to a deterministic approach of fitting the Cole-Cole model, please see Chen et al. (2008). We considered uniform prior distributions for all parameters except for the low-frequency asymptote of the resistivity $\rho_{0}$, which we set to the resistivity value observed at the lowest measurement frequency of $1 \mathrm{mHz}$. Experimenting with the width of these prior distributions, we found that it had essentially no influence on the final result and only a relatively minor influence on the speed of convergence, which points to the inherent robustness of the inversion procedure.

Figure 3 shows a representative log-log plot of the observed phase as a function of the frequency from one of our SIP measurements. The corresponding saturated quartz sand sample was measured three times up and down the considered frequency range and clearly displays the resonance or relaxation effect described through the Cole-Cole model given by Eq. (1). For the considered data set, the resonance peak is located at $\sim 1 \mathrm{~Hz}$. At $\sim 50 \mathrm{~Hz}$ the graph shows some erratic noise, which is most likely related to the local power supply. For comparison, we also show the corresponding measurements for the saturating pore fluid only, which in this case was water with an electrical conductivity of $\sim 60 \mu \mathrm{S} / \mathrm{cm}$.

To get a feeling for the data errors associated with the SIP measurements, which must be taken into account in the McMC stochastic inversion procedure, we consider the error characteristics provided for the impedance-meter (Zimmerman et al. (2008), along with the results of repeated measurements that we have made on a number of different samples. Figure 3 shows measurements that were repeated six times on one of the SIP samples (sand F36). Clearly the results are consistent, and the random measurement errors can be seen to be very small. This is of course dependent upon the length of time that has lapsed between measurements, as the SIP readings will be affected by temperature, carbon dioxide levels, and any reactions occurring inside the cylinder. Systematic errors are to be expected for large lag times between measurements. Overall, we found that data quality is a significant issue for SIP measurements on weakly polarizable samples. In particular, we have noticed that our SIP measurements were adversely affected in the lower frequency range between $\sim 0.001$ and $0.01 \mathrm{~Hz}$. Although similar observations have been made by other researchers (Jougnot, personal communication, 2009; Okay, personal communication, 2009), the causes of these noise problems remain enigmatic. As a consequence, SIP measurements on the more coarse-grained samples, whose relaxation peaks are expected to be located at the lower end of the considered frequency range, must probably be regarded as representing the limits of current measurement capabilities. In this context, it is, however, important to note that due to the use of multiple repeated measurements over the entire frequency range and variable electrical conductivities of the saturating pore fluid, meaningful quantitative interpretations were, unless explicitly mentioned otherwise, possible for most of our samples.

\section{Results}

\subsection{Hydraulic characterization}

Figure 4 shows a semi-log plot of the saturated hydraulic conductivity versus porosity for the samples considered in this study. The samples are distinguished in terms of being compacted, non-compacted, sieved, and non-sieved. As expected, we observe that the compaction of a sample generally results in a reduced hydraulic conductivity and porosity due to smaller pore diameters. Changing of the grain size distributions through the use of sieved fractions, on the other hand, shows a tendency towards greater porosity values for 


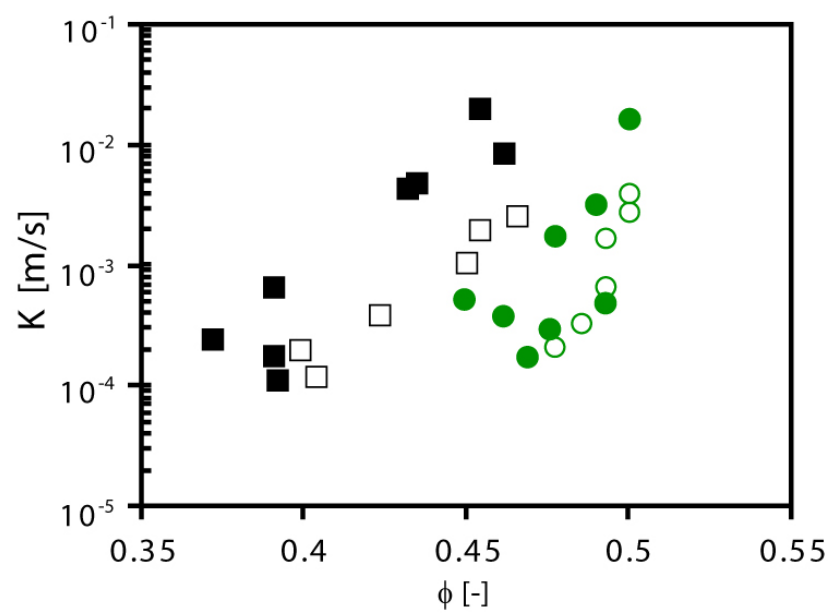

Fig. 4. Plot of the saturated hydraulic conductivity $K$ versus porosity $\phi$ for all of the samples considered in this study. Black squares represent compacted and unsieved samples, while full green circles represent uncompacted and unsieved samples. Sieved, well sorted sands are represented as non-solid black squares for compacted, and non-solid green circles for uncompacted samples.

comparable values of the hydraulic conductivities. At similar porosity, well-sorted samples tend to drain less efficiently than their more heterogeneous counterparts, and in other words need more pore space to conduct the same amount of water. It is assumed that pore size distribution is more homogeneous in well-sorted samples, and discrepancies between smallest and biggest pore sizes are smaller than with materials of broad grain size distributions. The above finding is consistent with the fact that a squared increase of the pore surface area, which governs frictional properties, is opposed by a cubic gain in pore volume, which governs the transport volume. Meaning that one pore with the same volume as two smaller pores combined imposes less friction on the water flux and hence transports more water in the same time. Although this statement seems to be largely self-evident and a principal factor in the formation of preferential, channelized flow patterns, this effect is rarely considered in current hydrological research (e.g., Hillel, 2004).

Figure 5 shows a semi-log plot of the saturated hydraulic conductivity versus the specific surface area. The specific surface area was evaluated with a Beckman Coulter LSTM 13320 Laser Diffraction Particle Size Analyzer. The method is applicable for non-compacted samples with grain diameters smaller than $2 \mathrm{~mm}$ and hence the total amount of measureable samples is somewhat limited. Nevertheless, the corresponding results demonstrate that well sorted samples show a much stronger and clearer correlation between hydraulic conductivity and specific surface area compared to their more poorly sorted, more heterogeneous counterparts. Overall, we see that for comparable surface areas, well sorted samples are characterized by higher hydraulic conductivity

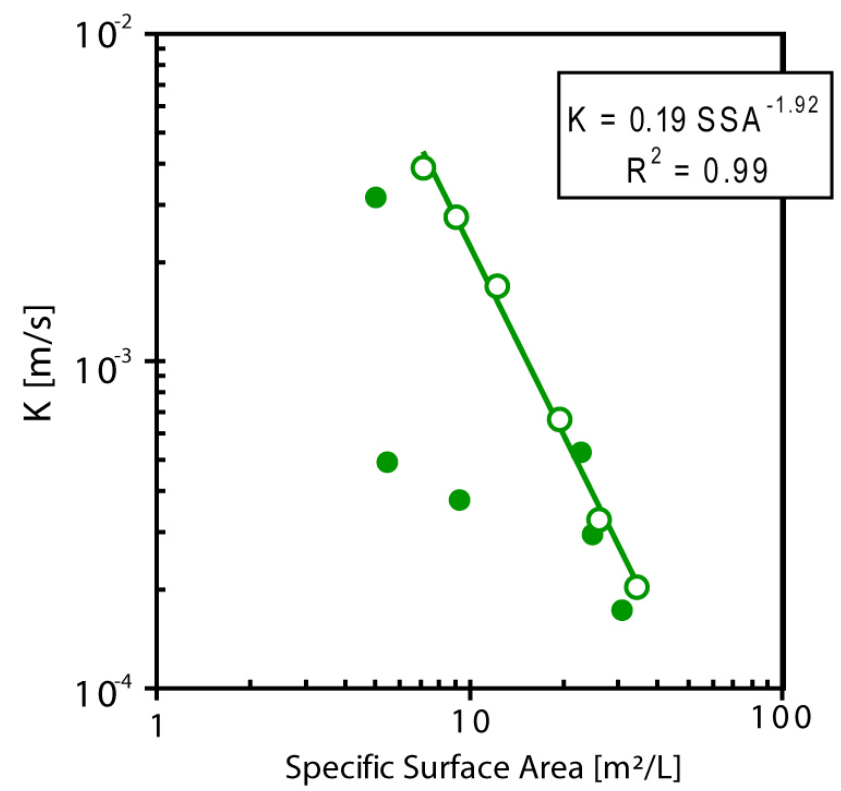

Fig. 5. Plot of the saturated hydraulic conductivity $K$ versus specific surface area (SSA) for non-compacted samples having grain sizes smaller than $2 \mathrm{~mm}$. Green solid circles denote unsieved samples, while non-solid green circles represent sieved sand fractions. The power law fit refers only to the data from sieved sand fractions.

than most of the more poorly sorted samples. Keeping in mind the above mentioned basic relationship of surface to volume, this discrepancy between sieved and non-sieved sands should be entirely related to the heterogeneity of the samples' pore structure. As a consequence, the discrepancy between the maximum value of the specific surface area for a certain value of the hydraulic conductivity and the actually observed value for a given sample should provide us with a parameter related to the actual width of a samples pore size distribution. This parameter is related to the dynamic storage and hydraulic transport behavior of a material and might be of use in hydrological modeling. In the considered case, the maximum values are given by the results for the well sorted samples, but could also be inferred through theoretical considerations.

\subsection{SIP measurements}

Figure 6 shows a log-log plot of the saturated hydraulic conductivity versus the estimated relaxation time $\tau$ obtained by inverting the measured SIP data based on the Cole-Cole model given by Eq. (1). Only compacted samples are considered and the least squares fitting was applied separately to the results for sieved and non-sieved samples. The latter exhibit a power law relation between the hydraulic conductivity, $K$, and the relaxation time, tau. The correlation between $K$ and $\tau$ is much stronger for the non-sieved samples than for the sieved samples, as a possible consequence of the relatively 


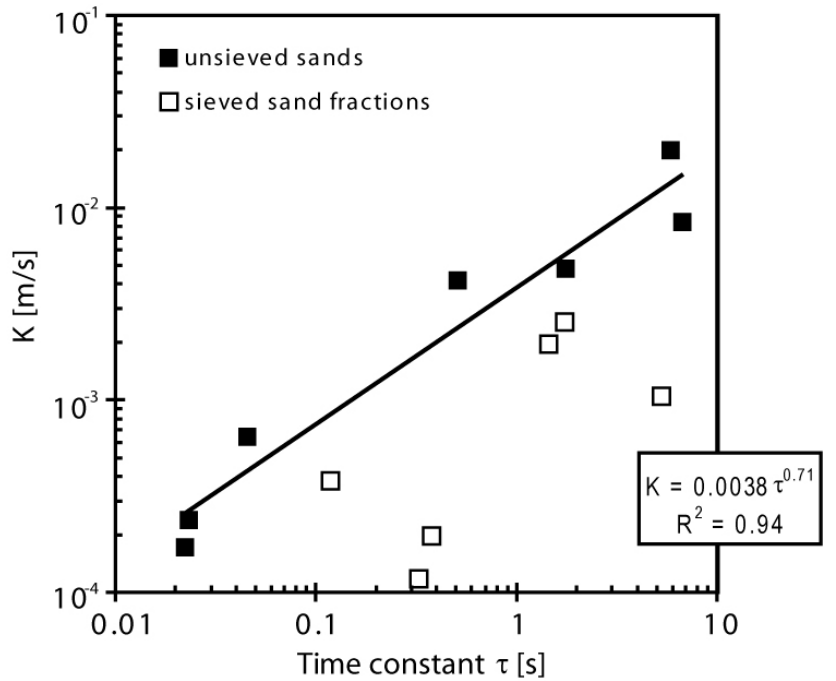

Fig. 6. Plot of the saturated hydraulic conductivity $K$ versus ColeCole relaxation time $\tau$ for sieved and unsieved samples using a conductivity of $\sim 300 \mu \mathrm{S} / \mathrm{cm}$ for the saturating pore water. In both cases the samples were compacted. The power law fit refers only to the data from unsieved sands, which is displayed as black full squares.

poor quality of the SIP measurements for the latter. This finding, which has been corroborated through repeated measurements, is enigmatic when interpreting the SIP-response based on the simple sphere model from Schwarz (1962). When considering the grain diameter to be one of the most decisive parameters we would expect to see a trend towards sharper, more clear, relaxation peaks, along with increasing granulometric homogeneity of the samples, which indirectly should result in a more clear $K-\tau$ correlation for better sorted sand.

Figure 7 shows a log-log plot of the saturated hydraulic conductivity versus the estimated Cole-Cole relaxation time, where compacted or non-compacted samples have now been separated. With regard to time constant $\tau$, the overall effect of compaction seems to be a shift towards smaller time constants, associated with smaller length scales of the underlying polarization process (Schwarz, 1962; Kormiltsev, 1963). The primary effect of compaction is the reduction of the porosity but likewise it results in increasing a sample's specific surface area per unit pore volume: for example, decreasing the pore space between uniform spheres by $10 \%$ through compaction results in an increase of the specific surface area per unit pore volume of almost $17 \%$. This demonstrates that together with the well known sensitivity of the SIP response to changes in grain size (e.g., Schwartz, 1962; Leroy et al., 2008; Revil and Florsch, 2010), the specific surface area per unit pore volume also seems to be a good indicator of the size of the polarization cells sensed by the inferred relaxation processes (e.g., Kormiltsev, 1963; Börner and Schön, 1991).

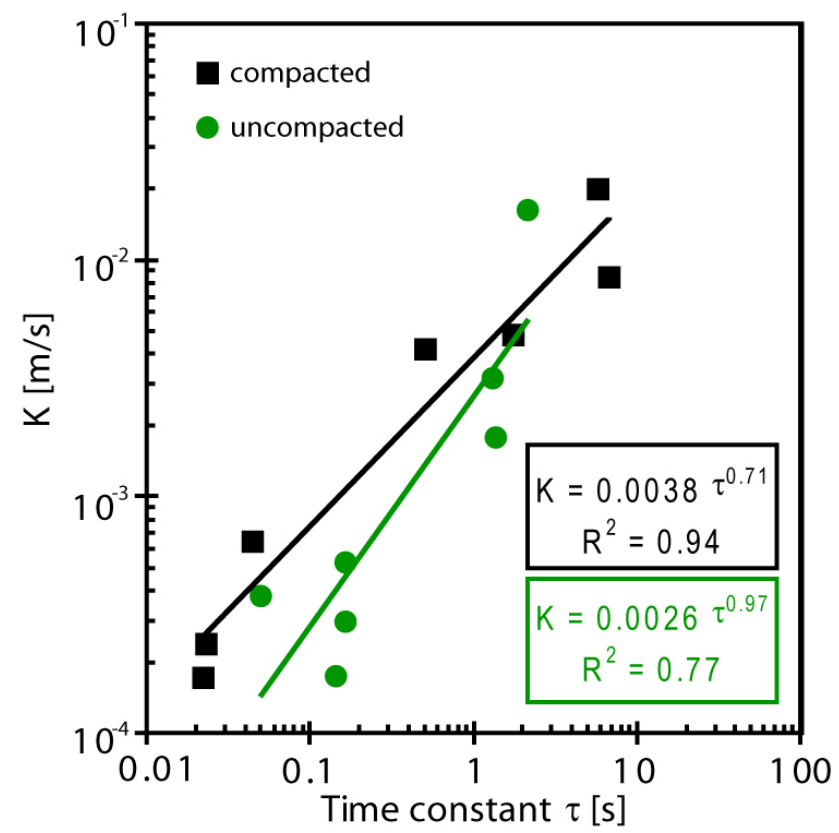

Fig. 7. Plot of the saturated hydraulic conductivity $K$ versus ColeCole relaxation time $\tau$ for compacted and uncompacted, unsieved, samples measured at $\sim 300 \mu \mathrm{S} / \mathrm{cm}$ electrical conductivities of the saturating pore water.

Finally, Fig. 8 compares the observed $K$-values and the ones inferred based on common granulometric models (Hazen, 1892; Beyer, 1964) with the $\tau$-values of the corresponding samples. Interestingly, we find a systematically stronger correlation for the measured $K$-values compared to the $K$-values inferred from empirical relations. In this context, it is interesting and important to note that these relationships are based on well sorted sand samples comparable to the ones considered here. Hence, the fact that the time constant data fit the actual measurements of hydraulic conductivity more consistently than granulometrically-based estimates suggests that the phenomenology of the SIP response cannot be reduced to a single textural factor, such as, for example, the grain size. As a consequence, this is once again an indication that consideration of multiple structural parameters will be necessary when trying to identify parameters governing the source processes of induced polarization. Please note that the values of all data points shown in Fig. 8, including those of the apparent outlier in the grain-size-based estimates with the smallest time constant, have been confirmed by repeated measurements.

In this context, Binley et al. (2005) pointed out that the Cole-Cole model time constant appears to be better correlated with $K$ than with other measures of the interfacial surface, such as, for example, the surface area per unit pore volume. In their work, values of the time constant are compared to median grain size $d_{50}\left(r^{2}=0.62\right.$; as compared to $r^{2}=0.64$ in our study (graph not shown)), pore throat diameter $\left(r^{2}=\right.$ 


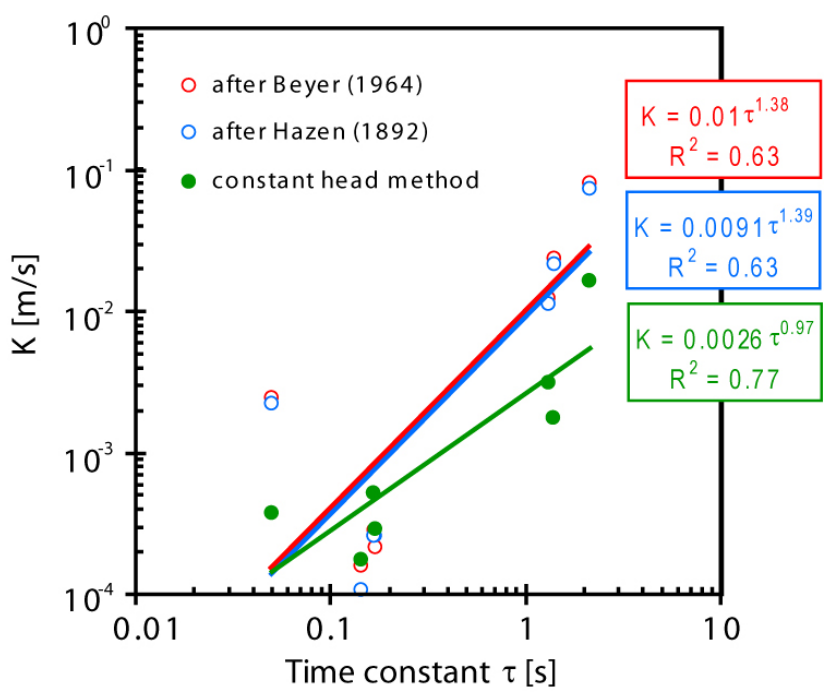

Fig. 8. Plot of the saturated hydraulic conductivity $K$ versus the Cole-Cole relaxation time $\tau$ for uncompacted samples measured at an electrical conductivity of the the saturating pore water of $\sim 60 \mu \mathrm{S} / \mathrm{cm}$. $K$ either was calculated from the grain-size distribution measurements using the empirical, grain-size-based formulas of Beyer (1964) and Hazen (1892) or experimentally determined through constant-head-type measurements.

$0.61)$, surface area per unit volume $\left(r^{2}=0.75\right)$, and hydraulic conductivity $\left(r^{2}=0.78\right.$; compared to $r^{2}=0.77$ for uncompacted samples and $r^{2}=0.94$ for compacted samples in our study (Figs. 6, 7)). Although these findings by Binley et al. (2005) are for consolidated sandstone samples, the results are in qualitative agreement with our findings. However, results shown in Figs. 4 and 5 indicate that changes in the sorting have a direct impact on these relationships. This is a further indication of the actual complexity of petrophysical parameter relationships and the processes which dominate hydraulic conductivity as well as induced polarization measures.

Following Reppert and Morgan (2001), Scott (2006), and Revil and Florsch (2010), the impact of the pore width on the recorded SIP response can be considered of secondary importance. This is primarily due to the comparatively large sizes of the pore throats of both non-compacted and compacted samples considered in this study, which in turn points to the generally subordinate contribution of pore throat and membrane effects to the observed SIP response for unconsolidated sandy sediments in the frequency range below the Maxwell-Wagner effect. At the same time, the observed impact on a samples' SIP-signature related to the transformation of pore space through compaction clearly indicates that individual structural aspects like grain size or surface roughness (e.g., Leroy et al., 2008) are not the only parameters determining SIP response. Compaction changes the specific surface area per unit pore volume, which in turn is expected to find its distinct expression in corresponding changes of the formation factor. As a consequence, the recently proposed theoretical model from Revil and Florsch (2010), in which the formation factor plays an essential role, seems to be indirectly supported by our findings. In detail, however, the link between the formation factor and the SIP response of granular sandy media is as of yet largely unexplored and, in our view, represents an important topic for future research in this domain.

\section{Conclusions}

The goal of this study was to improve our understanding of the polarization processes through experimental control of different pore space characteristics in laboratory measurements. Results show a clear relation of the SIP response with the hydraulic conductivity, demonstrate the variability of this relation in response to changes in pore size and grain size characteristics, and hence demonstrate potential of SIPbased methods for remote-sensing-type first-order hydraulic characterizations of the shallow subsurface. In agreement with previous findings, the results of our measurements indicate a power-law-type correlation between the inferred ColeCole relaxation time and hydraulic conductivity for the considered broad range of saturated sand samples. Changes in compaction and sorting of the samples resulted in a certain shift in the SIP response but did not fundamentally alter this overall picture. With regard to an improved understanding of the underlying physical properties, the strong interdependencies between grain size distribution and pore characteristics complicate the inference of the origin of the corresponding SIP effect. Yet, increasing the degree of compaction while leaving the grain size distribution unchanged showed a systematic effect on the SIP response towards smaller relaxation times, which in turn are associated with lower degrees of heterogeneity in the probed material. The phenomenological approach of relating values of the relaxation time to a change in the size of heterogeneities in the sampled material showed for both changes in grain size and pore size of our samples, while the change of pore size through compaction also effects the ratio pore interface versus porosity. Due to this interlink of specific surface area with compaction and pore width, multiple explanations are possible and hence fundamental question regarding the interdependencies of the polarization spectra and pore width remains as of yet unresolved.

Acknowledgements. This research has been funded by a grant from the Swiss National Science Foundation. We would like to thank the four reviewers André Revil, Lee Slater, and Maosong Tong, as well as an anonymous reviewer, for their constructive comments which helped to improve the manuscript. We greatly profited from collaboration with and logistical support from the Forschungszentrum Juelich, Germany. We would also like to thank Quarzwerke Frechen for providing the samples used for this study. Andreas Kemna acknowledges support by the SFB/TR32 "Patterns in 
soil-vegetation-atmosphere systems: Monitoring, modeling, and data assimilation" funded by the German Science Foundation (DFG).

Edited by: N. Romano

\section{References}

Beyer, W.: Zur Bestimmung der Wasserdurchlässigkeit von Kiesen und Sanden aus der Kornverteilung, Wasserwirtschaft Wassertechnik (WWT), 6, 165-169, 1964.

Binley, A., Slater L., Fukes M., and Cassiani, G.: The relationship between spectral induced polarization and hydraulic properties of saturated and unsaturated sandstone, Water Resour. Res., 41(12), W12417, doi:10.1029/2005WR004202, 2005.

Binley, A., Kruschwitz, S., Lesmes, D., and Kettridge, N.: Exploiting the temperature effects on low frequency electrical spectra of sandstone: a comparison of effective diffusion path lengths, Geophysics, 75(6), A43-A46, doi:10.1190/1.3483815, 2010.

Börner, F. D. and Schön, J. H.: A relation between the quadrature component of electrical conductivity and the specific surface area of sedimentary rocks, The Log Analyst, 32, 612-613, 1991.

Börner, F. D., Schopper, J. R., and Weller, A.: Evaluation of transport and storage properties in the soil and groundwater zone from induced polarization measurements, Geophysical Prospecting, 44, 583-601, 1996.

Chen, Y. and Or, D.: Effects of Maxwell-Wagner polarization on soil complex dielectric permittivity under variable temperature and electrical conductivity, Water Resour. Res., 42, W06424, doi:10.1029/2005WR004590, 2006.

Chen, J., Kemna, A., and Hubbard, S.: A comparison between Gauss-Newton and Markov-chain-Monte Carlo-based methods for inverting spectral induced-polarization data for Cole-Cole parameters, Geophysics, 73, F247-F259, doi:10.1190/1.2976115, 2008

Cole, K. S. and Cole, R. H.: Dispersion and absorption in dielectrics. I. alternating current field, J. Chem. Phys., 1, 341-351, 1941.

Cosenza, P., Ghorbani, A., Camerlynck, C., Rejiba, F., Guérin, R., and Tabbagh, A.: Effective medium theories for modelling the relationships between electromagnetic properties and hydrological variables in geomaterials: a review, Near Surf. Geophys., 7, 563-578, doi:10.3997/1873-0604.2009009, 2009.

Dias, C. A.: Developments in a model to describe low-frequency electrical polarization of rocks, Geophysics, 65, 43-451, 2000.

Dukhin, S. S. and Shilov, V. N.: Dielectric Phenomena and the Double Layer in Dispersed Systems and Polyelectrolytes, John Wiley and Sons: (http://pubs.acs.org/appl/literatum/publisher/achs/ journals/entities/2009.gif) New York, 1974.

Hazen, A.: Some physical properties of sands and gravels, with special reference to their use in filtration, 24th Annual Rep., Massachusetts State Board of Health, 34, 539-556, 1892.

Hillel, D.: Introduction to environmental soil physics, Elsevier, 494 pp., 2004.

Holliger, K.: Groundwater geophysics: from structure and porosity to permeability?, in: Overexploitation and Contamination of Shared Groundwater Resources, edited by: Darnault, C., Springer, Dordrecht, The Netherlands, 49-65, 2008.
Hördt, A., Blaschek, R., Kemna, A., and Zisser, N.: Hydraulic conductivity estimation from induced polarisation data at the field scale - the Krauthausen case history, J. Appl. Geophys., 62, 33 46, 2007.

Jougnot, D., Ghorbiani, A., Revil, A., Leroy, P., and Cosenza, P.: Spectral induced polarization of partially saturated clay-rocks: a mechanistic approach, Geophys. J. Int., 180, 210-224, 2010.

Kemna, A., Binley, A., and Slater, L.: Crosshole IP imaging for engineering and environmental applications, Geophysics, 69, 97107, 2004.

Kemna, A., Münch, H.-M., Titov, K., Zimmermann, E., and Vereecken, H.: Relation of SIP relaxation time of sands to salinity, grain size and hydraulic conductivity: Extended Abstracts: Near Surface 2005 - 11th European Meeting of Environmental and Engineering Geophysics, 4 pp., 2005.

Koch, K., Wenninger, J., Uhlenbrook, S., and Bonell, M.: Joint interpretation of hydrological and geophysical data: electrical resistivity tomography results from a process hydrological research site in the Black Forest Mountains, Germany, Hydrol. Process., 23(10), 1501-1513, 2009.

Kormiltsev, V. V.: O vozbuzdenii i spade vyzvannoi polarizatsii v kapillarnoi srede (On excitation and decay of induced polarization in capillary medium): Izvestia AN SSSR, Seria Geofizicheskaya (Solid Earth Physics), 11, 1658-1666, 1963 (in Russian).

Kruschwitz, S., Binley, A., Lesmes, D., and Elshenawy, A.: Textural controls on low- frequency spectra of porous media, Geophysics, 75, 113-123, doi:10.1190/1.3479835, 2010.

Leroy, P., Revil, A., Altmann, S., and Tournassat, C.: Modeling the composition of the pore water in a clay-rock geological formation(Callovo-Oxfordian, France), Geochim. Cosmochim. Acta, 71(5), 1087-1097, doi:10.1016/j.gca.2006.11.009, 2007.

Leroy, P., Revil, A., Kemna, A., Cosenza, P., and Ghorbani, A.: Complex conductivity of water-saturated packs of glass beads, J. Colloid Interf. Sci., 321, 103-117, 2008.

Leroy, P. and Revil, A.: A mechanistic model for the spectral induced polarization of clay material, J. geophys. Res., 114, B10202, doi:10.1029/2008JB006114, 2009.

Lesmes, D. P. and Morgan, F. D.: Dielectric spectroscopy of sedimentary rocks, J. Geophys. Res., 106, 13329-13346, 2001.

Lesmes, D. P. and Friedman, S.: Relationships between electrical and hydrogeological properties of rocks and soils, in: Hydrogeophysics, edited by: Rubin and Hubbard, Springer, Dordrecht, The Netherlands, 87-128, 2005.

Marshall, D. J. and Madden, T. R.: Induced polarization, a study of its causes, Geophysics, 24, 790-816, 1959.

Maxwell, J. C.: A Treatise on Electricity and Magnetism, third ed., Oxford University Press, London, 1892.

Mosegaard, K. and Tarantola, A.: Monte Carlo sampling of solutions to inverse problems, J. Geophys. Res., 100, 12431-12447, doi:10.1029/94JB03097, 1995.

Pape, H. and Vogelsang, D.: Fractal evaluation of induced polarization logs in the KTB-Oberpfalz HB, Geologisches Jahrbuch, Bundesanst. für Geowiss. u. Rohstoffe, E 54, 3-27, 1996.

Pelton, W. H., Ward S. H., Hallof, P. G., Sill, W. R., and Nelson, P. H.: Mineral discrimination and removal of inductive coupling with multifrequency IP, Geophysics, 43, 588-609, 1978.

Reppert, P. M. and Morgan, F. D.: Streaming potential data collection and data processing techniques, J. Colloid Interf. Sci., 233, 348-355, 2001. 
Revil, A. and Glover, P. W. J.: Theory of ionic-surface electrical conduction in porous media, Physical Review B, 55(3), 17571773, 1997.

Revil, A. and Florsch, N.: Determination of permeability from spectral induced polarization in granular media, Geophys. J. Int., 181(3), 1480-1498, doi:10.1111/j.1365-246X.2010.04573.x, 2010.

Rubin, Y. and Hubbard, S. (Eds.): Hydrogeophysics, Springer, Dordrecht, The Netherlands, 523 pp., 2005.

Scott, J. B. T.: The origin of the observed low-frequency electrical polarization in sandstones, Geophysics, 71, 235-238, 2006.

Schmutz, M., Revil, A., Vaudelet, P., Batzle, M., Femenía Viñao, P., and Werkema, D. D.: Influence of oil saturation upon spectral induced polarization of oil bearing sands, Geophys. J. Int., 183, 211-224, doi:10.1111/j.1365-246X.2010.04751.x, 2010.

Schwarz, G.: A theory of the low-frequency dispersion of colloidal particles in electrolyte solution, J. Phys. Chem., 66, 2636-2642, 1962.

Slater, L. and Lesmes, D. P.: Electrical-hydraulic relationships observed for unconsolidated sediments, Water Resour. Res., 38(10), 1213, doi:10.1029/2001WR001075, 2002.

Slater, L.: Near surface electrical characterization of hydraulic conductivity: from petrophysical properties to aquifer geometries a review, Surv. Geophys., 28, 169-197, 2007.

Stern, O.: Zur Theorie der elektrolytischen Doppelschicht, Z. Electrochem, 30, 508, 1924.

Titov, K., Komarov, V., Tarasov, V., and Levitski, A.: Theoretical and experimental study of time-domain induced polarization in water saturated sands, J. Appl. Geophys., 50, 417-433, 2002.
Titov, K., Kemna, A., Tarasov, A., and Vereecken, H.: Induced polarization of unsaturated sands determined through time domain measurements, Vadose Zone J., 3, 1160-1168, 2004.

Titov, K., Tarasov, A. Ilyin, Y., Seleznev, N., and Boyd, A.: Relationships between induced polarization relaxation time and hydraulic properties of sandstone, Geophys. J. Int., 180, 10951106, 2010.

Tong, M., Li, L., Wang, W., and Jiang, Y.: A time-domain inducedpolarization method for estimating permeability in a shaly sand reservoir, Geophys. Prosp., 54, 623-631, 2006.

Vanhala, H.: Mapping oil-contaminated sand and till with the spectral induced polarization (SIP) method, Geophys. Prosp., 45, 303-326, 1997.

Vinegar, H. J. and Waxman, M. H.: Induced polarization of shaly sands, Geophysics, 49, 1267-1287, 1984.

Wagner, K. W.: Erklärung der dielektrischen Nachwirkungsvorgänge auf Grund der Maxwellschen Vorstellungen, Arch. Elektrotechn, 2, 371-387, 1914.

Zimmermann, E., Kemna, A., Berwix, J., Glaas, W., Münch, H. M., and Huisman, J. A.: A high-accuracy impedance spectrometer for measuring sediments with low polarizability, Meas. Sci. Technol., 19, 105603, doi:10.1088/0957-0233/19/10/105603, 2008.

Zisser, N., Kemna, A., and Nover, G.: Relationship between low-frequency electrical properties and hydraulic permeability of low-permeability sandstones, Geophysics, 75, E131-E141, 2010. 